\title{
What's for lunch on Enceladus? How oxidant production generates a rich metabolic menu
}

\author{
CHRISTINE RAY ${ }^{1,2}$, CHRISTOPHER GLEIN $^{2}$, J. HUNTER \\ WAITE JR. ${ }^{1,2}$, BEN TEOLIS ${ }^{2}$, TORI HOEHLER ${ }^{3}$, JULIE A \\ HUBER $^{4}$, JONATHAN LUNINE ${ }^{5}$ AND FRANK POSTBERG ${ }^{6}$ \\ ${ }^{1}$ University of Texas at San Antonio \\ ${ }^{2}$ Southwest Research Institute \\ ${ }^{3}$ NASA Ames Research Center \\ ${ }^{4}$ Woods Hole Oceanographic Institute \\ ${ }^{5}$ Cornell University \\ ${ }^{6}$ Freie Universität Berlin \\ Presenting Author: christine.ray@contractor.swri.org
}

The mass spectrometer onboard the Cassini spacecraft detected molecular hydrogen $\left(\mathrm{H}_{2}\right), \mathrm{CO}_{2}$, and methane $\left(\mathrm{CH}_{4}\right)$ in the plume of Saturn's moon Enceladus. These measurements indicate that there is free energy for methanogenesis, the metabolic reaction of $\mathrm{H}_{2}$ with $\mathrm{CO}_{2}$ to form $\mathrm{CH}_{4}$ and water, in Enceladus' ocean [1]. Other metabolisms could also serve as energy sources in the ocean, but require oxidants that were not detected in the plume. To constrain the concentrations of these oxidants and determine their impact on energy availability, we present a model of oxidant production and redox chemistry on Enceladus [2]. We estimate the radiolytic production rates of $\mathrm{O}_{2}$ and $\mathrm{H}_{2} \mathrm{O}_{2}$ in the ice shell and ocean, and consider three cases for how these oxidants may contribute to Enceladus' overall oxidant budget, either through accumulation in the ocean, or through conversion into $\mathrm{SO}_{4}^{2-}$ and ferric oxyhydroxides by aqueous reductants and/or seafloor minerals. We use Cassini observations of volatiles [1,3], major ions [4,5], and organic compounds [6] in the plume as model constraints. For all cases, we determine upper limits on the concentrations and production rates of oxidants, and calculate available energy for key metabolic reactions. We compare our free energy estimates with requirements for microbes on Earth, and find that both aerobic and anaerobic metabolic reactions could meet these requirements in all considered cases. Additionally, we use these estimates to calculate microbial cell densities that could hypothetically be sustained on Enceladus, and compare these numbers to cell densities in Earth environments. We show that aerobic metabolisms could sustain up to $\sim 1$ cell $\mathrm{cm}^{-3}$ within a $20 \mathrm{~m}$ depth across Enceladus' seafloor, and that anaerobic metabolisms could sustain up to $\sim 1$ cell for every two $\mathrm{cm}^{-3}$ within this volume. Our results indicate that oxidant production and oxidation chemistry could contribute to supporting metabolically diverse microbial life on Enceladus.

[1] Waite et al. (2017) Science, 356, 155-159 [2] Ray et al. (2020), Icarus, 114248. [3] Magee and Waite (2017), LPS XLVIII, 2974 [4] Postberg et al. (2009), Nature, 459(7250), 1098-110 [5] Postberg et al. (2011), Nature, 474(7353), 620-622 [6] Postberg et al. (2018), Nature, 558(7711), 564-568 